Short Communication

\title{
Chromosomal localization of microsatellite loci in Drosophila mediopunctata
}

\author{
Renato Cavasini, Marcos Roberto Dias Batista and Louis Bernard Klaczko \\ Departamento de Genética, Evolução e Bioagentes, Instituto de Biologia, \\ Universidade Estadual de Campinas, Campinas, SP, Brazil.
}

\begin{abstract}
Drosophila mediopunctata has been used as a model organism for genetics and evolutionary studies in the last three decades. A linkage map with 48 microsatellite loci recently published for this species showed five syntenic groups, which had their homology determined to Drosophila melanogaster chromosomes. Then, by inference, each of the groups was associated with one of the five major chromosomes of $D$. mediopunctata. Our objective was to carry out a genetic (chromosomal) analysis to increase the number of available loci with known chromosomal location. We made a simultaneous analysis of visible mutant phenotypes and microsatellite genotypes in a backcross of a standard strain and a mutant strain, which had each major autosome marked. Hence, we could establish the chromosomal location of seventeen loci; including one from each of the five major linkage groups previously published, and twelve new loci. Our results were congruent with the previous location and they open new possibilities to future work integrating microsatellites, chromosomal inversions, and genetic determinants of physiological and morphological variation.
\end{abstract}

Keywords: tripunctata, SSR, genetic chromosomal analysis, BLAST search, Muller's elements.

Received: September 21, 2014; Accepted: November 23, 2014.

Chromosomal localization of genetic markers is an essential tool for the identification of factors associated to biological traits (Carvalho and Klaczko, 1993; Cazemajor et al., 1997; Noor et al., 2000; Hatadani et al., 2004). Several traits are mapped using microsatellite loci, for example: growth rates (Laine et al., 2013; Ori et al., 2014), behaviour (Oxley et al., 2010; Laine et al., 2014), courtship song (Schäfer et al., 2010; Lagisz et al., 2012), viability (Plough, 2012), male sterility (Moriguchi et al., 2014), pathogen resistance (Stephens et al., 2014), among others. Furthermore, physical and linkage maps may allow insights about evolutionary events that occurred in natural populations and genomic rearrangement within a specific line (Barker et al., 2009; Santos et al., 2010; Simões et al., 2012).

Drosophila mediopunctata is a forest dwelling Neotropical species that has the typical "primitive" Drosophila karyotype with five pairs of rods and a pair of dot chromosomes (Kastritsis, 1966). In the last three decades, this species has been used as a model organism for genetics and evolutionary studies (Klaczko, 2006) that characterized chromosomal inversion polymorphism (Peixoto and Klaczko, 1991; Ananina et al., 2004; Batista et al., 2012), morphological variation (Klaczko and Bitner-Mathé, 1990;

Send correspondence to Louis Bernard Klaczko. Departamento de Genética, Evolução e Bioagentes, Instituto de Biologia, Universidade Estadual de Campinas, 13083-970 Campinas, SP, Brazil. E-mail: Ibk@unicamp.br.
Andrade et al., 2005) and their association (Bitner-Mathé et al., 1995; Hatadani et al., 2004; Andrade et al., 2009).

A total of 134 microsatellite loci markers were described for D. mediopunctata (Laborda et al., 2009). A linkage map with five syntenic groups for 48 loci was published (Laborda et al., 2012). Each of these groups was associated to one of the five major D. mediopunctata chromosomes by cross-reference to BLAST searches on the $D$. melanogaster genome (a $49^{\text {th }}$ locus was assigned to the dot chromosome). The synteny between $D$. mediopunctata and D. melanogaster chromosomes was established using fluorescence in situ hybridization (FISH) in polytene chromosomes of highly conserved genes (Brianti et al., 2013). An analysis of the Drosophila 12 Genomes Consortium (2007) data showed that the chromosomes in this genus are highly conserved (Bhutkar et al., 2008); 95\% of about 13,000 D. melanogaster genes are located on the same Muller element across the twelve species. Thus, the use of BLAST searches to locate a marker is reliable, but a small fraction of markers remains without a completely certain location. Usually, a threshold value for the BLAST e-value in the range of $10^{-3}$ to $10^{-5}$ is frequently used to assign homology; thus $10^{-5}$ represents a conservative approach for multiple comparisons (Domazet-Loso and Tautz, 2003). Therefore, our main objective is to increase the number of available loci assigned to chromosomes.

We performed a chromosomal localization of D. mediopunctata microsatellite loci using a genetic (chro- 
mosomal) analysis. Three inbred strains were used. Strain CR27A carries mutations with visible effects marking each of the major autosomes: it is heterozygous for the dominant visible and recessive lethal mutation Delta-5 $(\Delta-5)$, located on chromosome II; and it is homozygous for recessive mutations cabernet ( $c b)$, coral ( $c r)$ and alfinete ( $a l)$, located on chromosomes III, IV and V, respectively. CR27B shares with CR27A the same genetic background, except for chromosome II, where it is homozygous for the wild type chromosome of CR27A. The other strain (ITA24P) has no marker mutations (wild type alleles for the mutations mentioned above). A description of the strains and the crossing outline are shown in detail by Carvalho and Klaczko (1993) and Hatadani et al. (2004).

Briefly, we crossed a CR27A male with an ITA24P virgin female in vials with trimeveledon culture medium (Rocha et al., 2009) at $18{ }^{\circ} \mathrm{C}$. Every other day, the couple was transferred to a new vial for up to six transfers. The analysis of the first generation flies (three F1 males and three F1 females) allowed the identification of sex-linked microsatellite loci by the inheritance pattern (heterozygous females and hemizygous males for X-linked loci). Autosomal microsatellite loci were located analyzing the backcross offspring of an F1 male with a CR27B virgin female. Since there is no recombination in males of D. mediopunctata (Cavasini et al., 2010), the microsatellite alleles of CR27A strain always segregate with their syntenic visible mutation (microsatellite locus and visible mutation on the same chromosome). A sample of 48 backcross offsprings was used for this analysis, three individuals from each of the 16 possible phenotypes - permutations of four (mutation or wild) phenotypes. Figure 1 shows an example of this procedure.

The genomic DNA extraction followed the protocol described by Aljanabi and Martinez (1997) with modifications. Amplification of microsatellite loci followed the parameters described in Cavasini et al. (2013). Polymerase Chain Reaction (PCR) products were all firstly visualized in $1 \%$ agarose gels stained with SYBR ${ }^{\circledR}$ Safe DNA gel staining (Invitrogen); thereafter, if necessary, they were also revealed in $6 \%$ polyacrylamide gels stained with $\mathrm{AgNO}_{3}$. Silver staining procedures followed the method described by Creste et al. (2001) with modifications.

A total of 36 loci were tested, 17 of which were located (Table 1). It was not possible to locate the other loci due to the presence of shared alleles by the strains used in the crosses. Five loci were used as controls since they were already listed in the linkage map previously published by Laborda et al. (2012). Each one is located in one of the five corresponding syntenic groups established by the linkage map, and respectively on the five major D. mediopunctata chromosomes. Therefore, our results were congruent with the previous localization established by homology, thus, confirming the location of 49 loci tested by Laborda et al. (2012). In addition, 12 loci were added, totaling 61

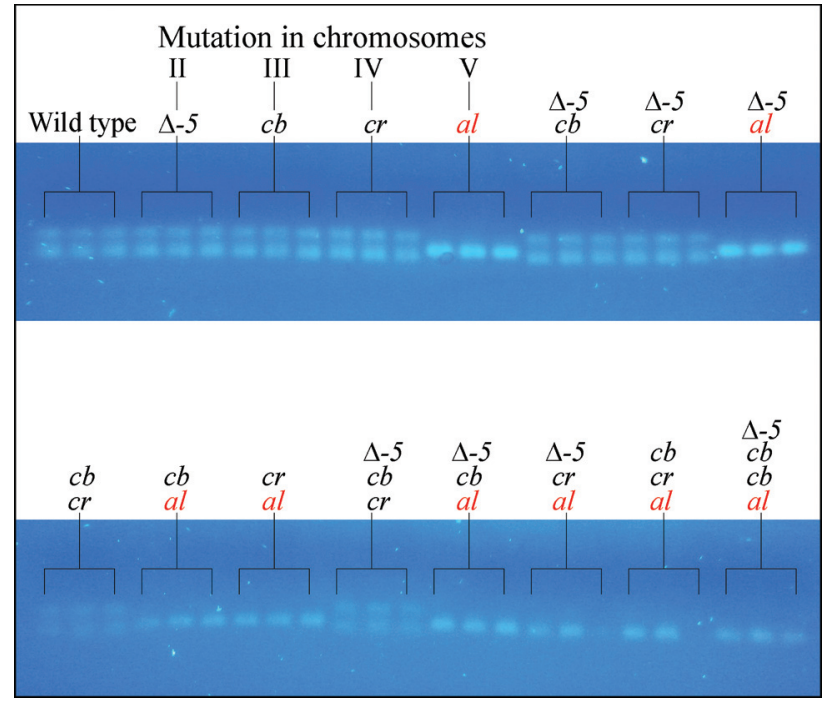

Figure 1 - Sample of 48 backcross offsprings genotyped for Dmed $^{\text {UNICAMP }}$ ssr011 locus (three individuals per phenotype). Mutations Delta-5 ( $\Delta-5)$, cabernet $(c b)$, coral $(c r)$ and alfinete $(a l)$, are located on chromosomes II, III, IV and V, respectively. All the backcross offsprings that express the recessive mutation alfinete (in red) are homozygous for all loci in chromosome V, such as the Dmed UNICAMP__ssr011 locus. On the other hand, all the backcross offsprings that do not express alfinete are heterozygous for all loci in chromosome $\mathrm{V}$, with different alleles in the parental strains.

microsatellite loci with their chromosomal location confirmed. Since CR27A carries no visible marker on the dot chromosome (VI), there is a very low probability $(3.6 \mathrm{x}$ $10^{-15}$ ) of mapping any of 15 autosomal loci on dot chromosome (for a similar procedure see Noor et al., 2000).

Table 1 shows BLAST results (E-values) of D. mediopunctata sequences matched to the D. melanogaster genome. The first BLAST column shows E-values matched to the respective D. melanogaster homologous chromosome. The second BLAST column shows the smallest E-value matched to a non-homologous D. melanogaster chromosome. Thirteen loci showed E-values consistent to their actual chromosomal location. Nevertheless, the loci Dmed ${ }^{\text {UNICAMP }}$ ssr084 and

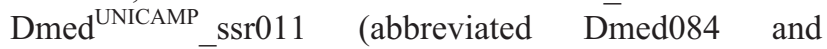
Dmed011, respectively, and similarly henceforward) showed inconclusive BLAST results, with the same Evalue for different chromosomes. Nonetheless, our analysis located them unambiguously on chromosomes III and V, respectively. For the Dmed094 and Dmed131 loci, the BLAST results $\left(0.177\right.$ and $\left.6.7 \times 10^{-5}\right)$ point out, respectively, non-significantly to chromosome $\mathrm{V}$ and misleadingly to the X chromosome; yet, in fact, both loci are on chromosome IV. Thus, although the gene content in Muller's elements is highly conserved in Drosophila, some small sequences may not be easily assigned to homologous chromosomes in other species. In these cases, the genetic (chromosomal) analysis is a powerful tool to elucidate their locations. 
Table 1 - Chromosomal localization of 17 microsatellites loci of $D$. mediopunctata by synteny with visible phenotype of mutations (autosomes) or by sex-linked inheritance pattern (sex chromosome).

\begin{tabular}{|c|c|c|c|c|}
\hline CHR & Mutation & Locus & BLAST $^{b}$ & BLAST $^{\circ}$ \\
\hline \multirow[t]{2}{*}{ X } & & Dmed021 & $9.4 \times 10^{-23}$ & 0.073 \\
\hline & & Dmed113 ${ }^{a}$ & $6.1 \times 10^{-10}$ & 2.142 \\
\hline \multirow[t]{3}{*}{ II } & Delta-5 $(\Delta-5)$ & Dmed018 & 0.005 & 0.019 \\
\hline & & Dmed060 & $1.0 \times 10^{-13}$ & 0.342 \\
\hline & & Dmed118 a & $1.2 \times 10^{-18}$ & 0.059 \\
\hline \multirow[t]{5}{*}{ III } & cabernet (cb) & Dmed067 & $2.5 \times 10^{-30}$ & 0.427 \\
\hline & & Dmed084 & 1.151 & 1.151 \\
\hline & & Dmed085 & $4.9 \times 10^{-22}$ & 1.487 \\
\hline & & Dmed095 & $2.2 \times 10^{-16}$ & 0.011 \\
\hline & & Dmed106 ${ }^{\text {a }}$ & 0.008 & 0.129 \\
\hline \multirow[t]{4}{*}{ IV } & $\operatorname{coral}(\mathrm{cr})$ & Dmed094 & 2.761 & 0.177 \\
\hline & & Dmed $102^{a}$ & $1.5 \times 10^{-21}$ & 0.071 \\
\hline & & Dmed103 & $2.3 \times 10^{-17}$ & 0.005 \\
\hline & & Dmed131 & 0.064 & $6.7 \times 10^{-5}$ \\
\hline \multirow[t]{3}{*}{ V } & alfinete (al) & Dmed011 ${ }^{a}$ & 0.210 & 0.210 \\
\hline & & Dmed025 & $7.1 \times 10^{-20}$ & 0.001 \\
\hline & & Dmed072 & $1.7 \times 10^{-11}$ & 0.059 \\
\hline
\end{tabular}

CHR stands for chromosome.

Loci names are abbreviated, such as Dmed021 for Dmed ${ }^{\text {UNICAMP }}$ ssr021. ${ }^{a}$ Loci present in the linkage map published by Laborda et al. (2012).

${ }^{\mathrm{b}}$ BLAST results (E-values) of D. mediopunctata sequences matched to $D$. melanogaster homologous chromosome.

${ }^{c}$ BLAST results (smallest E-value) of D. mediopunctata sequences matched to D. melanogaster non-homologous chromosome.

\section{Acknowledgments}

We are grateful to Claudete do Couto, Klélia A. Carvalho and Mitsue T. Brianti for technical assistance. Funds were provided by: Fundação de Amparo à Pesquisa do Estado de São Paulo (FAPESP); Conselho Nacional de Desenvolvimento Científico e Tecnológico (CNPq); Coordenação de Aperfeiçoamento de Pessoal de Ensino Superior (CAPES); and Fundo de Apoio ao Ensino e à Pesquisa (FAEPEX-UNICAMP).

\section{References}

Aljanabi SM and Martinez I (1997) Universal and rapid saltextraction of high quality genomic DNA for PCR-based techniques. Nucleic Acids Res 25:4692-4693.

Ananina G, Peixoto AA, Bitner-Mathé BC, Souza WN, Silva LB, Valente VLS and Klaczko LB (2004) Chromosomal inversion polymorphism in Drosophila mediopunctata: Seasonal, altitudinal, and latitudinal variation. Genet Mol Biol 27:6169.

Andrade CAC, Hatadani LM and Klaczko LB (2005) Phenotypic plasticity of the aedeagus of Drosophila mediopunctata: Effect of the temperature. J Therm Biol 30:518-523.
Andrade CAC, Vieira RD, Ananina G and Klaczko LB (2009) Evolution of the male genitalia: Morphological variation of the aedeagi in a natural population of Drosophila mediopunctata. Genetica 135:13-23.

Barker JSF, Frydenberg J, González J, Davies HI, Ruiz A, Sorensen JG and Loeschcke V (2009) Bottlenecks, population differentiation and apparent selection at microsatellite loci in Australian Drosophila buzzatii. Heredity 102:389-401.

Batista MRD, Ananina G and Klaczko LB (2012) Unexpected long-term changes in chromosome inversion frequencies in a Neotropical Drosophila species. Clim Res 53:131-140.

Bhutkar A, Schaeffer SW, Russo SM, Xu M, Smith TF and Gelbart WM (2008) Chromosomal rearrangement inferred from comparisons of 12 Drosophila genomes. Genetics 179:1657-1680.

Bitner-Mathé BC, Peixoto AA and Klaczko LB (1995) Morphological variation in a natural population of Drosophila mediopunctata: Altitudinal cline, temporal changes and influence of chromosome inversions. Heredity 75:54-61.

Brianti MT, Ananina G and Klaczko LB (2013) Differential occurrence of chromosome inversion polymorphisms among Muller's elements in three species of the tripunctata group of Drosophila, including a species with fast chromosomal evolution. Genome 56:17-26.

Carvalho AB and Klaczko LB (1993) Autosomal suppressors of sex-ratio in Drosophila mediopunctata. Heredity 71:546551.

Cavasini R, Carvalho KA and Klaczko LB (2010) Absence of recombination in males of Drosophila mediopunctata. Dros Inf Serv 93:122-124.

Cavasini R, Batista MRD, Carvalho KA and Klaczko LB (2013) Optimization of 64 microsatellite loci primer pair annealing temperatures of Drosophila mediopunctata. Dros Inf Serv 96:218-219.

Cazemajor M, Landré C and Montchamp-Moreau C (1997) The Sex-Ratio trait in Drosophila simulans: Genetic analysis of distortion and suppression. Genetics 147:635-642.

Creste S, Neto AT and Figueira A (2001) Detection of single sequence repeat polymorphisms in denaturing polyacrylamide sequencing gels by silver staining. Plant Mol Biol Rep 19:299-306.

Domazet-Loso T and Tautz D (2003) An evolutionary analysis of orphan genes in Drosophila. Genome Res 13:2213-2219.

Drosophila 12 Genomes Consortium (2007) Evolution of genes and genomes on the Drosophila phylogeny. Nature 450:203-218.

Hatadani LM, Baptista JCR, Souza WN and Klaczko LB (2004) Colour polymorphism in Drosophila mediopunctata: Genetic (chromosomal) analysis and nonrandom association with chromosome inversions. Heredity 93:525-534.

Kastritsis CD (1966) Cytological studies of some species of the tripunctata group of Drosophila. Univ Texas Publs Stud Genet 6615:413-474.

Klaczko LB (2006) Evolutionary genetics of Drosophila mediopunctata. Genetica 126:43-55.

Klaczko LB and Bitner-Mathé BC (1990) On the edge of a wing. Nature 346:321.

Laborda PR, Mori GM and Souza AP (2009) Drosophila mediopunctata microsatellites I: More than a hundred polymorphic loci available for genetic studies. Conserv Genet Resour 1:297-307. 
Laborda PR, Gazaffi R, Garcia AAF and Souza AP (2012) A molecular linkage map for Drosophila mediopunctata confirms synteny with Drosophila melanogaster and suggests a region that controls the variation in the number of abdominal spots. Insect Mol Biol 21:89-95.

Lagisz M, Wen S-Y, Routtu J, Klappert K, Mazzi D, MoralesHojas R, Schäfer MA, Vieira J, Hoikkala A, Ritchie MG and Butlin RK (2012) Two distinct genomic regions, harbouring the period and fruitless genes, affect male courtship song in Drosophila montana. Heredity 108:602-608.

Laine VN, Shikano T, Herczeg G, Vilkki J and Merilä J (2013) Quantitative trait loci for growth and body size in the ninespined stickleback Pungitius pungitius L. Mol Ecol 22:5861-5876.

Laine VN, Herczeg G, Shikano T, Vilkki J and Merilä J (2014) QTL analysis of behavior in nine-spined sticklebacks (Pungitius pungitius). Behav Genet 44:77-88.

Moriguchi Y, Ueno S, Higuchi Y, Miyajima D, Itoo S, Futamura N, Shinohara K and Tsumura Y (2014) Establishment of a microsatellite panel covering the sugi (Cryptomeria japonica) genome, and its application for localization of a malesterile gene ( $m s-2)$. Mol Breed 33:315-325.

Noor MAF, Schug MD and Aquadro CF (2000) Microsatellite variation in populations of Drosophila pseudoobscura and Drosophila persimilis. Genet Res 75:25-35.

Ori RJ, Esmailizadeh AK, Charati H, Mohammadabadi MR and Sohrabi SS (2014) Identification of QTL for live weight and growth rate using DNA markers on chromosome 3 in an $F_{2}$ population of Japanese quail. Mol Biol Rep 41:1049-1057.

Oxley PR, Spivak M and Oldroyd BP (2010) Six quantitative trait loci influence task thresholds for hygienic behaviour in honeybees (Apis mellifera). Mol Ecol 19:1452-1461.
Peixoto AA and Klaczko LB (1991) Linkage disequilibrium analysis of chromosomal inversion polymorphisms of Drosophila. Genetics 129:773-777.

Plough LV (2012) Environmental stress increases selection against and dominance of deleterious mutations in inbred families of the Pacific oyster Crassostrea gigas. Mol Ecol 21:3974-3987.

Rocha F, Medeiros HF and Klaczko LB (2009) The reaction norm for abdominal pigmentation and its curve in Drosophila mediopunctata depend on the mean phenotypic value. Evolution 63:280-287.

Santos J, Serra L, Solé E and Pascual M (2010) FISH mapping of microsatellite loci from Drosophila subobscura and its comparison to related species. Chromosome Res 18:213-226.

Schäfer MA, Mazzi D, Klappert K, Kauranen H, Vieira J, Hoikkala A, Ritchie MG and Schlötterer C (2010) A microsatellite linkage map for Drosophila montana shows large variation in recombination rates, and a courtship song trait maps to an area of low recombination. J Evol Biol 23:518-527.

Simões P, Calabria G, Picão-Osório J, Balanyà J and Pascual M (2012) The genetic content of chromosomal inversions across a wide latitudinal gradient. PLoS One 7:e51625.

Stephens A, Lombardi M, Cogan NOI, Forster JW, Hobson K, Materne M and Kaur S (2014) Genetic marker discovery, intraspecific linkage map construction and quantitative trait locus analysis of ascochyta blight resistance in chickpea (Cicer arietinum L.). Mol Breed 33:297-313.

Associate Editor: Klaus Hartfelder

License information: This is an open-access article distributed under the terms of the Creative Commons Attribution License, which permits unrestricted use, distribution, and reproduction in any medium, provided the original work is properly cited. 